\title{
Principaux effets psychiques des stupéfiants : risques en milieu professionnel
}

\section{Main psychophysiological effects of narcotic drugs : hazards in workplace}

Jean-Pierre ANGER*

Laboratoire de Toxicologie, Faculté de Pharmacie, Université de Rennes 1, 2, avenue du Professeur Léon Bernard - 35043 RENNES Cedex

* Auteur à qui adresser la correspondance : Jean-Pierre ANGER, Laboratoire de Toxicologie, Faculté de Pharmacie, Université de Rennes 1, 2, avenue du Professeur Léon Bernard - 35043 RENNES Cedex Tél : 0223234867 - e-mail : anger@univ-rennes1.fr

(Reçu le 1'er février 2002 ; accepté le 15 février 2002)

\section{$\boldsymbol{R E} \boldsymbol{E} U \boldsymbol{M E}$}

Parmi les psychotropes faisant l'objet de conduites addictives en milieu professionnel, outre l'alcool, le tabac ou les médicaments psycho-actifs, on rencontre parfois les stupéfiants illicites comme le cannabis, les opiacés, la cocaïne et les amphétaminiques. Ces substances sont utilisées soit pour mieux gérer son stress au travail ou à l'inverse se doper pour un meilleur rendement. Après absorption par différentes voies (pulmonaire, intra-nasale, orale ou parentérale), les drogues franchissent la barrière hémato-encéphalique et gagnent le cerveau dont elles vont perturber le fonctionnement en mimant l'action ou en bloquant la sécrétion ou enfin en empêchant la recapture de certains neuromédiateurs. Ainsi les opiacés inhibent sélectivement de nombreuses activités neuronales induites par des stimulis excitateurs si bien que l'information arrive au cerveau non seulement «en retard» mais considérablement amoindrie, voire déformée et les réactions sont également diminuées. La cocaïne et les

\section{SUMMARY}

Psychotropic drugs leading to addictive conduct in the work place include not only alcohol, tobacco or psychoactive drugs but also illicit drugs such as cannabis, opiates, cocaine and amphetamines. These substances are used to better manage one's stress at work or, on the contrary, to boost one's performance. After absorption by various routes (pulmonary, intra-nasal, oral or parenteral) these drugs cross the blood-brain barrier, reach the brain and disrupt its functions by mimicking the action, or by blocking secretion or at least by preventing the recapture of some neuromediators. Thus, as opiates selectively inhibit many neuronal activities induced by excitation stimuli, the information that gets to the brain is not only delayed but considerably weakened. The cocaïne and amphetamines are to reverse of powerful psychostimulants. These compounds inhibit recapture in the brain of biogen amines (adrenaline, dopamine, serotonin), which induces increased neurotransmission. Under their 
amphétaminiques sont à l'inverse de puissants psychostimulants. Ces composés inhibent, au niveau cérébral la recapture des amines biogènes (noradrénaline, dopamine, sérotonine) ce qui induit un accroissement de la neurotransmission. Sous leur influence, la vigilance augmente, le temps de réaction s'améliore. Cependant d'autres effets associés comme l'euphorie, l'agressivité, la fatigue liée à l'insomnie conduisent à des comportements parfois irrationnels et dangereux. D’une façon générale, le dysfonctionnement cérébral induit par la prise de ces stupéfiants modifie plus ou moins profondément le comportement du sujet qui ne sera plus en mesure de juger sainement une situation critique et pourra, en conséquence, soit sous estimer le risque ou au contraire aura tendance à augmenter la prise de risque et dans certaines circonstances à favoriser l'accident.

\section{MOTS-CLÉS}

Opiacés, cocaïne, amphétamines, effets psychophysiologiques, risques, milieu professionnel.

\section{Introduction}

Les stupéfiants sont des psychotropes ou substances psychoactives licites ou illicites capables de modifier le fonctionnement du cerveau et de générer une dépendance. On sait aujourd'hui que quel que soit le produit, son administration aboutit à augmenter l'activité des neurones dopaminergiques du cerveau. Ces neurones particuliers impliqués dans la genèse et le contrôle des émotions représentent une voie finale commune à l'action de la plupart des drogues. Cette convergence de l'effet des différents produits addictifs sur le système dopaminergique explique les sensations, en particulier le plaisir, éprouvées lors de la prise de la drogue, mais d'autres systèmes neuronaux sont également mis en jeu expliquant par là même qu'en dehors du plaisir, d'autres effets se manifestent et diffèrent selon le produit consommé. Dans cet article très général consacré à l'usage des drogues psychoactives illicites en milieu professionnel, nous nous limiterons à l'étude des effets psychiques des opiacés, de la cocaïne et des amphétamines. Après avoir rappelé le mécanisme d'action des drogues au niveau du cerveau et donné quelques informations toxicocinétiques sur leur durée d'action dans l'organisme, nous décrirons essentiellement les effets de ces stupéfiants sur le comportement en cas d'utilisation ponctuelle et/ou chronique au travail et tenterons ainsi de mieux comprendre les risques qu'ils peuvent occasionner.

\section{Rappel sur le mécanisme d'action général des drogues au niveau du cerveau $(1,6)$}

influence, vigilance increases and reaction time is shorter. However other associated effects such as euphoria, aggressiveness and tiredness linked to insomnia, sometimes lead to dangerous behaviours. In general brain dysfunction induced by taking these drugs has a more or less profound effect on the subject's behaviour. He will no longer be able to assess a critical situation soundly and will, as a result, either underestimate risks or on the contrary have a tendency to take more risks and in some circumstances that may increase the risk of accidents.

\section{KEY-WORDS \\ Opiates, cocaïne, amphetamines, psychophysiological effects, hazards, working place.}

Les substances toxicomanogènes ont pour cible principale le cerveau où elles agissent au niveau de la cellule neuronale et au niveau de structures spécialisées de l'encéphale. Les onze milliards de neurones du cerveau sont reliés les uns aux autres pour transmettre l'information sur le plan fonctionnel mais non sur le plan de la continuité anatomique. L'information est transmise le long du neurone comme une impulsion électrique, activité qui est peu affectée par la plupart des drogues toxicomanogènes. La connexion fonctionnelle entre les neurones est la synapse, lieu de largage des neurotransmetteurs qui acheminent l'information d'un neurone à l'autre. On connaît aujourd'hui une quarantaine de neuromédiateurs différents dans le cerveau. Certains stimulent l'activité cérébrale : ce sont les neurotransmetteurs de type adrénergique : noradrénaline (NA), dopamine (DA), sérotonine (5-HT) ; d'autres, au contraire l'atténuent : c'est le cas de l'acide gamma amino butyrique (GABA) ou des endorphines. Tous ces neuromédiateurs vont gérer notre comportement. Chacun d'eux est produit par un type particulier de neurone et, une fois libéré, peut atteindre d'autres neurones. Un neuromédiateur donné se fixe sur une cellule nerveuse par l'intermédiaire d'un récepteur. On peut comparer le neuromédiateur à une clé qui reconnaît une serrure particulière, le récepteur et l'ouvre. Chaque neuromédiateur possède un ou plusieurs récepteurs spécifiques. Ce système permet la coexistence d'une grande variété de circuits de communication cérébraux qui régissent notre comportement (humeur, faim, soif, sexualité, agressivité ou passivité, etc...). En temps normal, tous ces neuromédiateurs sont secrétés en quantité infime de telle sorte que l'ensemble de notre comportement se trouve dans un état d'équilibre harmonieux, c'est le bien-être ou état d'homéostasie cérébrale. 
Les drogues sont capables d'ouvrir ces serrures biochimiques et de bouleverser cet équilibre soit en mimant l'action, soit en bloquant la sécrétion ou enfin en empêchant la recapture de certains neurotransmetteurs. Ainsi les opiacés (héroïne, morphine, codéine) miment l'action des endorphines et entraînent analgésie et sédation. L'information arrive bien au cerveau mais «en retard» et considérablement amoindrie, voire déformée et les réactions sont également diminuées. À l'inverse, la cocaïne, les amphétamines inhibent au niveau cérébral la recapture des amines biogènes (NA, DA et 5-HT) ce qui induit un accroissement de la neurotransmission. Le cerveau est alors contraint de changer de régime et de maintenir pour fonctionner une production accrue de catécholamines qui sera entretenue par l'apport fréquent de cocaïne. D'où le comportement impulsif quasi obligatoire de consommation de cette drogue par le cocaïnomane.

Toutes ces stimulations vont gagner deux zones particulièrement réactives du cerveau profond :

- 1'hypothalamus (système limbique ou «cerveau des émotions»), centre d'intégration des sensations de satisfaction également impliqué dans les fonctions indispensables à la survie de l'espèce (faim, soif, sexualité) ;

- l'aire tegmentale ventrale (ATV ou système de récompense), source de production de dopamine dans le cerveau. La dopamine libérée stimule alors le noyau accumbens, aujourd'hui reconnu comme le centre du plaisir et de la récompense. Cette stimulation est donc à l'origine d'une récompense mais aussi de dépendance.

Le désir de consommer des psychotropes serait donc lié au plaisir qu'ils procurent ! Cette constatation est née des observations faites dans les années 90 sur des modèles animaux comme le rat ou le singe qui, placés dans certaines conditions, ont tendance à répéter la consommation de substances psychoactives. Grâce aux techniques de micro-dialyse qui permettent, à l'aide de micro-électrodes de mesurer et ainsi de connaître les variations de concentration en messagers chimiques au niveau d'une zone cérébrale précise, on a découvert l'existence d'un mécanisme d'action commun à tous les psychotropes (alcool, cannabis, nicotine, morphine, amphétamines), caractérisé par l'augmentation du taux de DA au niveau du noyau accumbens. Ainsi l'autoadministration permet à l'animal en appuyant sur un levier de s'administrer un produit par différentes voies. $\mathrm{Si}$ le produit active le système de récompense, l'animal va appuyer de manière répétitive sur le levier pour s'auto-administrer le produit. Ces expériences ont ainsi permis de déterminer les zones cérébrales impliquées dans le système qui gère le plaisir : le système de récompense ou système hédonique chez l'homme.
On comprend donc mieux aujourd'hui comment les drogues agissent sur notre cerveau et par là même sur notre comportement mais malheureusement, on ne détient pas encore la substance miracle qui permettrait de remettre tout en ordre.

\section{Principaux effets psycho- physiologiques des drogues Effets des opiacés $(2,4,6)$}

On regroupe sous le terme opiacés différents produits remarquables par la puissance de leur action pharmacologique. Ces produits sont soit des alcaloïdes naturels de l'opium (morphine, codéine), soit des dérivés hémisynthétiques (héroïne, codéthyline, pholcodine), soit enfin des composés totalement synthétiques (buprénorphine, fentanyl). L'emploi et la délivrance de ces substances peuvent être libres de prescription (codéine, codéthyline, pholcodine) ou soumis à la réglementation des stupéfiants (morphine, buprénorphine, méthadone) ou enfin illicites (héroïne).

L'héroïne passe très rapidement dans le cerveau où elle est transformée en morphine. La morphine comme tous les opiacés agit en se fixant sur divers types de récepteurs spécifiques (opiorécepteurs) en mimant l'action des endorphines, ce qui explique son action analgésique puissante contre les douleurs fortes ou rebelles. La morphine stimule également les systèmes de la dopamine mais par un mécanisme indirect en diminuant le contrôle négatif qu'exercent habituellement les neurones à GABA sur les neurones à dopamine.

Sur le plan toxicocinétique, rappelons que l'héroïne a une demi-vie sanguine très courte et qu'elle est très rapidement métabolisée en 6-monoacétylmorphine (6-MAM) puis en morphine qui sera éliminée principalement sous forme de glucuroconjugué durant plusieurs jours. La codéine et la codéthyline sont également transformées en morphine. La buprénorphine et la méthadone ne se dégradent pas en morphine.

Les effets psychiques des opiacés diffèrent selon leur utilisation.

En cas d'utilisation ponctuelle et à faible dose, on observe :

- une action analgésique accompagnée de sédation, somnolence et sommeil. La sédation de la douleur est due à l'augmentation du seuil de perception de celle-ci par suite de l'inhibition de la libération de substance $P$ (neuromédiateur spécifique des voies nerveuses contrôlant les sensations douloureuses) et de l'activation des systèmes inhibiteurs bloquant l'action des neurones de la douleur (voies ascendantes de la douleur). La mor- 
phine provoque donc un état d'indifférence aux stimulis douloureux ;

- une action dépressive des centres respiratoires pouvant conduire à des malaises ;

- une action psychodysleptique avec euphorie, voire dysphorie, exaltation de l'imagination et hallucinations ;

- une hypotension et une bradycardie diminuant les performances du myocarde sans modifier le débit ( d'ou un risque d'arrêt cardiaque brutal) ;

- une constipation liée à une diminution du tonus et du péristaltisme intestinal.

Les effets psychophysiologiques de l'héroïne sont identiques à ceux de la morphine mais étant plus liposoluble, cette dernière agit plus vite et plus intensément et la dépression respiratoire est plus marquée. On estime que son action est trois fois plus forte que celle de la morphine. À fortes doses, l'héroïne provoque des bouffées délirantes, hallucinatoires et des phases d'excitation motrice parfois convulsives. L'utilisation chronique d'héroïne peut entraîner une toxicomanie caractérisée par une dépendance physique et psychique ainsi que par une tolérance. Les troubles occasionnés peuvent rendre le sujet instable et plus ou moins dangereux pour les autres :

- perturbations continues du psychisme avec réactions paranoïaques ;

- grandes variations de l'état de conscience ;

- comportements asociaux, agressifs, dépressifs.

Tous ces effets induisent une perte de l'attention, des réflexes, de la réalité et de la conscience du danger. Leur utilisation est donc particulièrement contre-indiquée au cours de travaux nécessitant concentration et dextérité.

Par ailleurs, l'association héroïne et alcool est très dangereuse car elle augmente fortement la dépression centrale donc la sédation et la vigilance.

Les effets indésirables de la codéine sont comparables à ceux des autres opiacés mais plus modérés et plus rares. La pholcodine, dérivée de la codéine, possède une action encore plus faible. Leur association à l'alcool entraîne une forte majoration des effets dépresseurs et sédatifs.

Bien qu'elle entraîne un syndrome de sevrage beaucoup moins important avec une dépression respiratoire plus faible, ce qui justifie son emploi en traitement de substitution, la buprénorphine possède une action analgésique considérée 25 à 40 fois supérieures à celle de la morphine. Son association à d'autres psychotropes dépresseurs du système nerveux central (alcool, benzodiazépines) est formellement déconseillée car elle entraîne l'endormissement et peut également provo- quer des hallucinations.

La méthadone est un psychotrope également utilisé en traitement de substitution de la dépendance aux opiacés. Les effets sont comparables à ceux des autres opiacés, mais ses propriétés euphorisantes sont faibles. Son action dépressive sur les centres respiratoires est plus élevée que celle de la morphine. Son utilisation répétée lui confère un effet sédatif marqué ainsi que l'apparition de mouvements réflexes exacerbés et une altération de la perception visuelle; tous ces effets s'avérant peu compatibles avec une activité réclamant l'attention et la précision.

\section{Effets de la cocaïne $(3,5,6)$}

La cocaïne est un alcaloïde extrait des feuilles de coca. Elle existe le plus souvent sous forme de chlorhydrate soluble dans l'eau (neige). Son absorption peut se faire par voie nasale (sniffing) ou plus rarement intraveineuse. Elle existe également sous forme de base insoluble dans l'eau mais soluble dans les lipides : le crack qui est fumé et qui passe ainsi très rapidement du poumon dans le cerveau. L'utilisation de cette forme de cocaïne induit une dépendance rapide, plus forte que lorsque l'alcaloïde est simplement sniffé.

Dans les pays occidentaux, la cocaïne est essentiellement utilisée par voies parentérale et pulmonaire. L'injection intraveineuse développe un effet euphorisant en moins d'une minute et qui persiste environ un quart d'heure. L'inhalation de la drogue permet l'absorption de la cocaïne par la muqueuse nasale, sans passage pulmonaire mais l'activité de la drogue par cette voie est très réduite du fait d'un effet de premier passage hépatique détruisant 70 à $80 \%$ de la dose. En revanche l'inhalation de vapeurs de crack détermine des effets comparables à ceux de l'injection intraveineuse. Quelle que soit la voie d'administration, la cocaïne est rapidement dégradée au niveau hépatique et par les estérases plasmatiques en plusieurs métabolites éliminés par voie urinaire. La demi-vie d'élimination, et donc d'action, est courte : de 30 minutes à 1 heure 30 .

La cocaïne agit en empêchant la recapture présynaptique d'un certain nombre de neuromédiateurs, en particulier de la dopamine et dans une moindre mesure de la noradrénaline et de la sérotonine. Ce faisant, elle augmente la présence et donc l'effet de la dopamine dans les synapses au niveau du système limbique, responsable d'un effet euphorisant très important.

Les effets psychophysiologiques de la consommation de cocaïne résultent pour l'essentiel d'une stimulation intense du système nerveux sympathique. Ils sont variables selon le mode de consommation. L'utilisateur occasionnel ressent constamment les effets psychologiques d'euphorie, de bien-être, d'empathie et d'hyper- 
vigilance. Son activité psychique est accrue. Il est sujet à des insomnies. Lorsque les doses sont répétées sur une brève période, la fin de l'activité du produit se traduit par une anxiété inclinant à utiliser de nouveau la drogue. Si la dose utilisée est plus importante, le consommateur peut être en proie à une agitation psychomotrice intense accompagnée d'idées délirantes (sentiment de persécution, illusions sensorielles, amnésies). Des comportements violents ont été rapportés après injection de la drogue ou inhalation de crack. À la longue le consommateur devient sujet à une grande instabilité caractérielle (dysphorie) avec délire d'interprétation à forme paranoïde, attaques de panique, etc. associés à des troubles de la vigilance et de la concentration peu propices à la sérénité durant le travail.

\section{Effets des amphétamines et de l'ecstasy $(3,5,6)$}

L'amphétamine est le chef de file d'une famille de substances chimiquement et pharmacologiquement proches les unes des autres dont les effets sont identiques et voisins de ceux induits par l'usage de la cocaïne.

La métamphétamine est la molécule la plus puissante de ce groupe. Elle est commercialisée sous la dénomination «ice» ou «glass» du fait de l'aspect blanc transparent de ses cristaux et de «crank» ou de «crystal» pour la forme salifiée, très hydrosoluble. Il s'agit d'une drogue utilisée sous forme de prise ou injectée (forme salifiée), mais aussi par inhalation, comme le crack, dans une pipe ou une cigarette.

De nombreuses amphétamines ont été commercialisées comme médicaments stimulants dans les années 70 et certaines spécialités sont demeurées célèbres : Ortédrine ${ }^{\circledR}$, Maxiton ${ }^{\circledR}$, Benzédrine ${ }^{\circledR}$. Des dérivés un peu moins nombreux ont été commercialisés comme anorexigènes : Cafilon ${ }^{\circledR}$, Fringalor ${ }^{\circledR}$ : leur usage a donné souvent lieu à des abus et à des troubles comportementaux en utilisation chronique ; il est désormais strictement contrôlé.

L'ecstasy ou méthylènedioxymétamphétamine (MDMA) est une phényléthylamine dont l'usage comme psychostimulant ne cesse de se développer. L'ecstasy est vendue sous forme de comprimés ou de gélules contenant quelques $\mathrm{mg}$ à plus de $200 \mathrm{mg}$ de MDMA. D'autres produits apparentés sont également vendus sous la dénomination d'ecstasy : MDB, MDEA, MBDB, 2-CB, etc. contenant parfois diverses substances plus ou moins toxiques.

Les amphétamines sont des agonistes sympathomimétiques indirects. Elles exercent leur activité directement sur les neurones utilisant la noradrénaline et la dopamine comme neuromédiateurs ; certaines d'entre elles, notamment l'ecstasy et autres phényléthylamines) ont une action préférentielle et presque spécifique sur les neurones à sérotonine. Dans tous les cas les amphétamines agissent sur la libération de ces neuromédiateurs : elles les déplacent de leur site de stockage dans le neurone présynaptique et augmentent massivement leur libération dans la synapse en «vidangeant» littéralement les neurones de leur contenu en neuromédiateur et en bloquant leur système de recapture. Ces stimulants de l'éveil ont donc en commun la propriété d'épuiser les neurones, expliquant que les effets psychostimulants et anorexigènes sont temporaires et ne perdurent que pendant le temps où l'activité de certains réseaux de neurones du cerveau est amplifiée par le recrutement (sous l'effet de la drogue) de toute la quantité de neurotransmetteur disponible dans la cellule. L'ecstasy agit essentiellement sur les neurones sérotoninergiques, neuromédiateurs impliqués dans la régulation de l'humeur ainsi que dans le contrôle de l'impulsivité. Elle entraîne une libération massive de sérotonine, une inhibition de sa synthèse et un blocage de sa recapture par le neurone émetteur. Les états dépressifs qui suivent la prise de MDMA pourraient être associés à cette diminution de la concentration en sérotonine au niveau cérébral. De même, l'hyperthermie constituant l'une des conséquences les plus graves de l'usage du MDMA évoque le syndrome d'hypersérotoninergie décrit au décours de l'usage de certains antidépresseurs inhibiteurs de la recapture de la sérotonine (ISRS).

Les amphétamines réduisent le sommeil ou souvent l'empêchent totalement. Elles augmentent de façon temporaire la vigilance et limitent la sensation de fatigue. Ces manifestations temporaires sont suivies d'une phase d'abattement, avec irritabilité, dépression, lassitude et souvent agressivité. Les sujets qui recourent régulièrement aux amphétamines font état d'une activité maniaque, d'altérations du jugement, d'une augmentation de l'agressivité parfois accompagnée de passage à l'acte, d'un amaigrissement (action anorexigène) et de l'insomnie. Après une prise de l'ordre de $150 \mathrm{mg}$ de MDMA, beaucoup d'utilisateurs décrivent une période de désorientation durant environ 30 minutes avec parfois des mouvements de crispation spasmodique notamment des muscles de la mâchoire : c'est le trismus. Suit une période de stimuli euphorique de 3 à 6 heures où la communication avec autrui est subjectivement améliorée, caractérisée par l'abolition de la sensation de fatigue et des troubles de la mémoire. La drogue favorise les mouvements répétitifs rythmés par la musique et contribue à faire perdre la notion du temps. Cette phase précède un état d'épuisement et de dépression durant environ 8 heures qui, parfois mal supportés, peuvent incliner à utiliser d'autres psychotropes censés en limiter l'expression (cannabis, anxio- 
lytiques, antidépresseurs). Parfois des crises aiguës d'angoisse voire des attaques de panique avec réactions violentes peuvent se manifester. L'usage d'ecstasy altère les performances professionnelles ainsi que les performances routières notamment la fatigue insurmontable suivant la phase d'insomnie provoquée par la molécule.

\section{Conclusion}

Parmi les psychotropes faisant l'objet de conduites addictives en milieu professionnel, outre l'alcool, le tabac ou les médicaments psycho-actifs, on rencontre parfois les stupéfiants illicites comme le cannabis, les opiacés, la cocaïne et les amphétaminiques. Ces substances sont utilisées soit pour mieux gérer son stress au travail ou à l'inverse se doper pour un meilleur rendement. Après absorption par différentes voies (pulmonaire, intra-nasale, orale ou parentérale), les drogues franchissent la barrière hémato-encéphalique et gagnent le cerveau dont elles vont perturber le fonctionnement. Nos connaissances sur leur mécanisme d'action ont nettement progressé depuis une vingtaine d'années. On sait aujourd'hui que les drogues, selon leur nature, agissent en mimant l'action, en bloquant la sécrétion ou en empêchant la recapture de certains neuromédiateurs bouleversant ainsi complètement la biochimie du cerveau. Ce dysfonctionnement cérébral induit par la prise de stupéfiants altère plus ou moins profondément le comportement du sujet qui ne sera plus en mesure de juger sainement une situation critique et pourra, en conséquence, soit sous estimer le risque ou au contraire aura tendance à augmenter la prise de risque et, dans certaines circonstances, à favoriser l'accident.

\section{Références}

1. Nahas G. Neuropsycho-pharmacologie de la dépendance. In : Nahas G., Trouvé R. Toxicomanie Pharmacodépendance, Paris, Masson Ed, 1988 ; 15-26.

2. Pépin G., Chèze M. Les opiacés. In : Mura P. (coordinateur), Alcool, médicaments, stupéfiants et conduite automobile . Paris, Elsevier-Option Bio, 1999 ; 75-95.

3. Vinner E., Dehon B., Ghysel M-H., Lhermitte M. Les psychostimulants. In : Mura P.(coordinateur), Alcool, médicaments, stupéfiants et conduite automobile. Paris, Elsevier Option Bio, 1999 ; 97-128.

4. Pépin G. Opiacés et opioïdes. In : Kintz P.(coordinateur), Toxicologie et Pharmacologie Médicolégales. Paris, Elsevier-Option Bio, 1998 ; 335-430.

5. Richard D., Pirot S., Senon J-L. Toxicomanies : Opiacés, LSD, CocaÏne, Amphétamines, Cannabis. In :

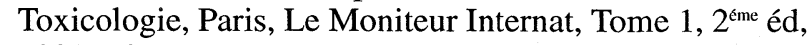
$1999 ; 277-311$.

6. Vasseur D., Chanson P. Drogues, savoir plus. Comité Français d'Education pour la Santé Paris, CFES, 1999 : $49 \mathrm{p}$. 